\title{
Viscoelastic Properties of UHMW-PE Fibers in Simple Elongation
}

\author{
P. J. R. LEBLANS and C. W. M. BASTIAANSEN, \\ DSM Research BV, Geleen, Netherlands, and L. E. GOVAERT, \\ T.U. Eindhoven, Netherlands
}

\begin{abstract}
Synopsis
Stress relaxation after a simple elongational step strain, creep under a constant simple elongational stress, and stress build-up under a constant Hencky strain rate have been measured for ultrahigh-molecular-weight polyethylene (UHMW-PE) fibers. The data from the various experiments are consistent with the Boltzmann superposition principle in the experimental region of small strains or short times. This leads to a simple constitutive equation in which temperature can be incorporated via time-temperature superpasition. The measured power-law relaxation of the UHMW-PE fiber leads to analytical expressions for the dynamic quantities in simple elongation.

The constitutive equation is the one-dimensional equivalent of the gel equation derived for crass-linking gels at the gel point. The similarity between the rheological behavior of fibers and cross-linking gels at the transition point might lead to an enhanced understanding of the molecular processes occurring during deformation.
\end{abstract}

\section{INTRODUCTION}

In the following, an attempt will be made to describe the viscoelastic properties of gelspun ultradrawn Ultra High-Molecular-Weight polyethylene (UHMW-PE) fibers (Dyneema SK 60) in uniaxial extension. A constitutive equation will be formulated for this fiber in the region of small deformations/short loading times. The temperature dependence of the mechanical properties will also be discussed.

\section{EXPERIMENTAL}

The UHMW-PE fiber used in this study was kindly supplied by Dyneema Vof, a joint venture of DSM (The Netherlands) and Toyobo (Japan). The multifilament yarn had a dtex which, assuming a density of $1000 \mathrm{~kg} / \mathrm{m}^{3}$, corresponds to a cross-sectional area of $1.66 \times 10^{-8} \mathrm{~m}^{2}$.

The mechanical properties of Dyneema SK 60 were measured on a Zwick 1474 tensile tester which was equipped with pneumatic fiber clamps and a thermostatically controlled oven. The use of fiber clamps introduces an uncertainty in the original length of the samples which was estimated to be about $5 \%$. The fibers were loaded to a stress of $10 \mathrm{MPa}$ before every experiment. A constant cross-head speed was used in the stress-strain measurements. This causes an error in the calculated strain rate which is negligible in the case of small strain. 


\section{MODELLING THE VISCOELASTIC PROPERTIES}

The following analysis is based on the observation that the stress relaxation modulus can be described with a power-law equation in the linear viscoelastic region as demonstrated in Figure 1 . The curves measured at $90^{\circ} \mathrm{C}$ that deviate from a straight line are considered to belong to the nonlinear viscoelastic region. The straight lines in the double-logarithmic plots have identical slopes $n$. The stress relaxation modulus $E$ is therefore

$$
E(t)=C t^{-n}
$$

where $n=0.072$ and $C=8.710^{10} \mathrm{~Pa}^{n} \mathrm{~s}^{n}$ at room temperature. Application of the Boltzmann superposition principle ${ }^{1}$ to simple extension of a viscoelastic liquid or solid leads to

$$
\sigma_{\mathrm{E}}(t)=\int_{-\infty}^{t} E\left(t-t^{\prime}\right) \dot{\epsilon}\left(t^{\prime}\right) d t^{\prime}
$$

where $\sigma_{\mathrm{E}}$ is the simple extensional stress, and $\dot{\epsilon}$ is the Hencky strain rate. The Hencky strain $k$ is defined as the natural logarithm of the ratio of the instantaneous length $l$ to the original length $l_{0}$, and $\dot{\epsilon}$ is its time derivative. Equation (2) is valid irrespective of whether the material has an equilibrium modulus. It must be emphasized that eq. (2) is only valid for simple extensional deformations. Since the fibers are neither incompressible nor isotropic, the equation cannot be translated directly to other deformation geometries. Also, eq. (2) is applicable only as long as the recoverable strain is kept within the linear region of the material.

The data shown in Figure 1 indicate that the extent of the linear region as defined here depends not only on the strain magnitude but also on the temperature. Various simple extensional experiments can now be analyzed

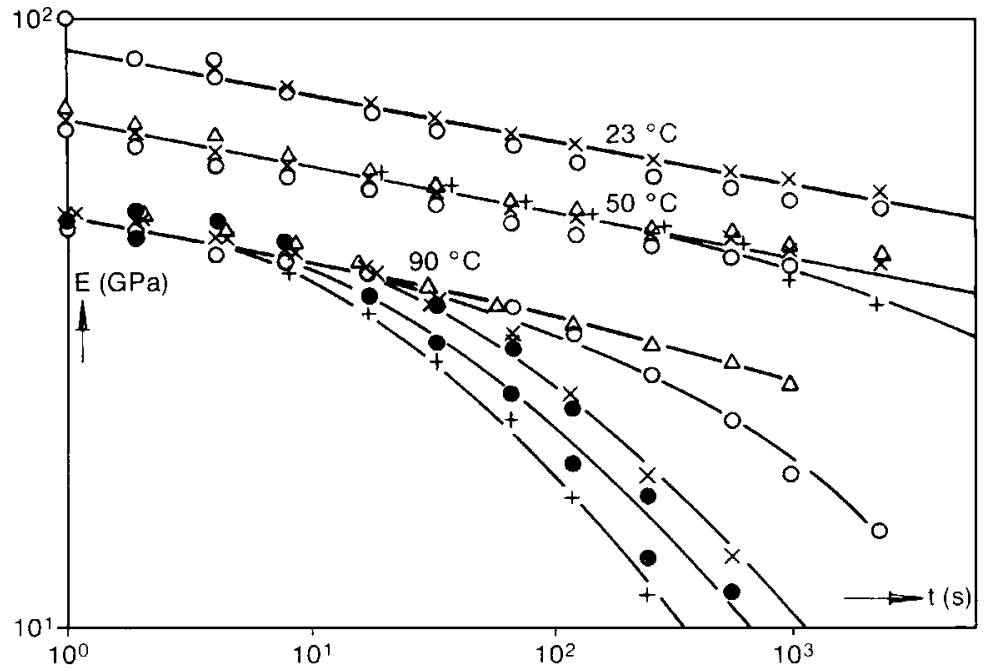

Fig. 1. Stress relaxation modulus measured at three different temperatures after steps in Hencky strain of $0.2 \%(\Delta), 0.4 \%(O), 1.0 \%(\times), 1.5 \%(\bullet), 2.0 \%(+)$. 
through the constitutive equation. The stress build-up at constant strain rate $\dot{\epsilon}$ can be calculated by combining eqs. (1) and (2):

$$
\sigma_{\mathrm{E}}(t)=\frac{\epsilon(t)}{1-n} E\left(\frac{\epsilon(t)}{\dot{\epsilon}}\right)=\frac{C}{1-n} \epsilon(t)^{1-n} \dot{\epsilon}^{n}
$$

The derivation of eq. (3) is based on the assumption that eq. (1) is valid for $0 \leq t \leq \infty$. This is obviously wrong for short times because it would imply that $\lim _{t \rightarrow 0} E=\infty$. The deviation from eq. (1) at short times will, however, have little influence on the predictions of the constitutive equation at longer times. Also, an equilibrium modulus might exist, but since no sign of it can be observed up to $t=10^{4} \mathrm{~s}$, it is believed to be of importance only for times much longer than $10^{4} \mathrm{~s}$. Consequently the introduction of an equilibrium modulus in the model has little influence on its predictions.

Double-logarithmic plots of $\sigma_{\mathrm{E}}$ versus the Hencky strain $\epsilon$ are therefore straight lines with slope $1-n$ and a level proportional to $\dot{\epsilon}^{-n}$. Figure 2 shows the lines calculated with eq. (3) together with the stress build-up under several Hencky strain rates. The constants $C$ and $n$ used in the calculations have been obtained from the stress relaxation measurements. The deviation of the calculated lines from the experimental points at high strains in Figure 2 is probably due to the fact that these points fall outside the range of applicability of the constitutive equation.

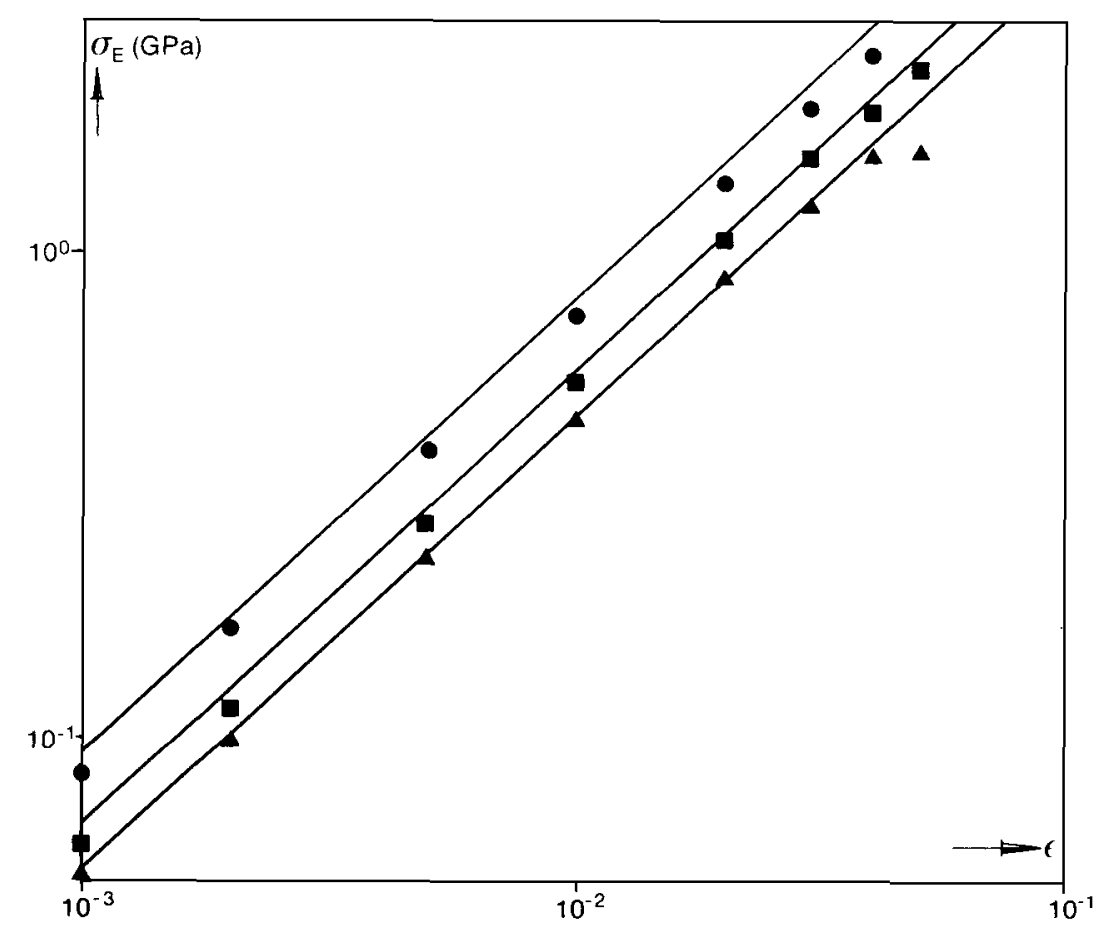

Fig. 2. Calculated (-) and measured stress build-up curves for Hencky strain rates of 8.4 $10^{-4} \mathrm{~s}^{-1}(\bullet), 8.410^{-6} \mathrm{~s}^{-1}(\square)$, and $4.210^{-7} \mathrm{~s}^{-1}(\nabla)$. 
According to eq. (3) the secant moduli $\left(E_{0.1 \%}, E_{0.5 \%}, \ldots\right)$ equal

$$
E_{\epsilon}=\frac{\sigma_{E}(\epsilon)}{\epsilon}=\frac{C}{1-n} \epsilon^{-n_{\dot{\epsilon}} n}
$$

Therefore, $E_{\epsilon}$ is proportional to $\dot{\epsilon}^{n}$ and inversely proportional to $\epsilon^{n}$. This dependence on the strain rate $\dot{\epsilon}$ was also observed by Porter for fibers produced by solid state extrusion of $\mathrm{HDPE}^{2}$ His data correspond to a power $n$ of 0.08 , which is close to the 0.072 found in this study. Analytical expressions are also obtained for the dynamic quantities when eqs. (1) and (2) are combined. The storage and loss moduli are ${ }^{3}$

$$
E^{\prime}(\omega)=\omega \int_{0}^{\infty} E(t) \sin \omega t d t=\frac{C \pi}{2 \Gamma(n) \sin \frac{(2-n) \pi}{2}} \omega^{n}
$$

and

$$
E^{\prime \prime}(\omega)=\omega \int_{0}^{\infty} E(t) \cos \omega t d t=\frac{C \pi}{2 \Gamma(n) \sin \frac{(1-n) \pi}{2}} \omega^{n}
$$

and the phase angle

$$
\delta=\operatorname{arctg} \frac{\sin [(2-n) \pi / 2]}{\sin [(1-n) \pi / 2]} \quad\left(\approx n \frac{\pi}{2} \text { for small values of } n\right)
$$

where $\Gamma(i)$ is the gamma function.

Capiati and Porter ${ }^{2}$ measured the storage modulus of fibers produced by solid-state extrusion of HDPE. It was proportional to $\omega^{x}$, where $x$ was roughly 0.06 . Because $\delta(\omega)$ is very sensitive to the shape of $E(t)$, the existence of an equilibrium modulus will have an influence on it. On the other hand eq. (7) will always predict well the order of magnitude of $\delta$ over a wide frequency range.

Partial integration of eq. (2) yields an equation with a memory function $m$ :

$$
\sigma_{\mathrm{E}}(t)=\int_{-\infty}^{t} m\left(t-t^{\prime}\right)\left[\epsilon(t)-\epsilon\left(t^{\prime}\right)\right] d t^{\prime}
$$

where $m(x)=-\partial E(x) / \partial x$. If $m$ is represented by a continuous spectrum of exponentially decaying terms,

$$
m\left(t-t^{\prime}\right)=\int_{-\infty}^{+\infty} \frac{H(\tau)}{\tau} e^{-\left(t-t^{\prime}\right) / \tau} d \ln \tau
$$

Equation (8) is identical with the (one-dimensional) Maxwell equation with a continuous relaxation time spectrum. In combination with eq. (1), $H(\tau)$ 
equals

$$
H(\tau)=\frac{C \tau^{-n}}{\Gamma(n)}
$$

From the relaxation time spectrum, the retardation time spectrum $L$ can be calculated ${ }^{3}$ :

$$
L(\tau)=\frac{\Gamma(n)}{\pi^{2} C\left(1+\operatorname{cotg}^{2} n \pi\right)} \tau^{n}
$$

which yields for the creep compliance

$$
D(t)=\frac{\Gamma(n) \Gamma(1-n)}{n \pi^{2} C\left(1+\operatorname{cotg}^{2} n \pi\right)} t^{n}+\frac{t}{\eta_{0 \mathrm{E}}}
$$

where $\eta_{0 E}$ is the zero-extension-rate viscosity, which equals

$$
\eta_{0 \mathrm{E}}=\int_{-\infty}^{+\infty} H(\tau) \tau d \ln \tau
$$

Introduction of the expression for $H$ given by eq. (10) into eq. (13) yields $\eta_{\mathrm{OE}}=\infty$. Furthermore, for the small value of $n$ found for the UHMW-PE fiber,

$$
\frac{\Gamma(n) \Gamma(1-n)}{n \pi^{2}\left(1+\operatorname{cotg}^{2} n \pi\right)} \simeq 1
$$

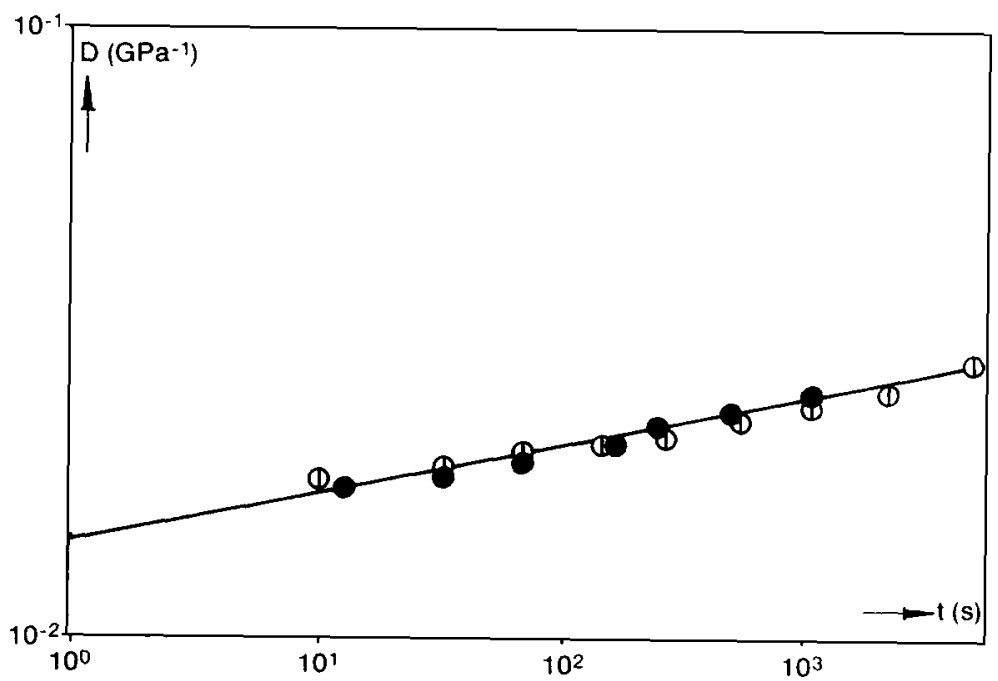

Fig. 3. Creep compliance at constant stresses of $0.4 \mathrm{GPa}(\Phi)$ and $0.8 \mathrm{GPa}(\bullet)$ at $50^{\circ} \mathrm{C}$ and $1 / E(t)(-)$. 
so that

$$
D(t) \simeq 1 / E(t)
$$

In the linear viscoelastic region this relation has been verified experimentally (Fig. 3).

\section{TIME-TEMPERATURE SUPERPOSITION}

Figure 1 shows stress relaxation moduli measured at different temperatures. In the linear viscoelastic region double-logarithmic plots with the same slope are measured at each temperature. Time-temperature superposition ${ }^{4}$ is therefore possible by shifting the curves along the $\log t$ axis. The idea behind this shift procedure is that the relaxation times of all relaxation mechanisms decrease by the same factor as the temperature increases. The temperature dependence can be incorporated into the constitutive equation by expressing the memory function $m$ as

$$
m\left(t-t^{\prime}, T\right)=\int_{-\infty}^{+\infty} \frac{H(\tau)}{a_{T_{0}}(T) \tau} e^{-\left(t-t^{\prime}\right) / a_{T_{0}}(T) \tau_{d \ln \tau}}
$$

where $T_{0}$ is the reference temperature, and $T$ is the measuring temperature. $\log a_{T_{0}}(T)$ is the distance between the double-logarithmic relaxation curves, measured at temperatures $T$ and $T_{0}$.

The existence of an equilibrium modulus of the same order of magnitude as the relaxing part of the modulus will complicate time-temperature superposition. Figure 4 shows the master curve of the stress relaxation modulus at room temperature, constructed from measuring data at 23,50 , and $90^{\circ} \mathrm{C}$ and a Hencky strain of $0.1 \%$. The curves shift over roughly 1.5 decades of time for a temperature difference of $20^{\circ} \mathrm{C}$.

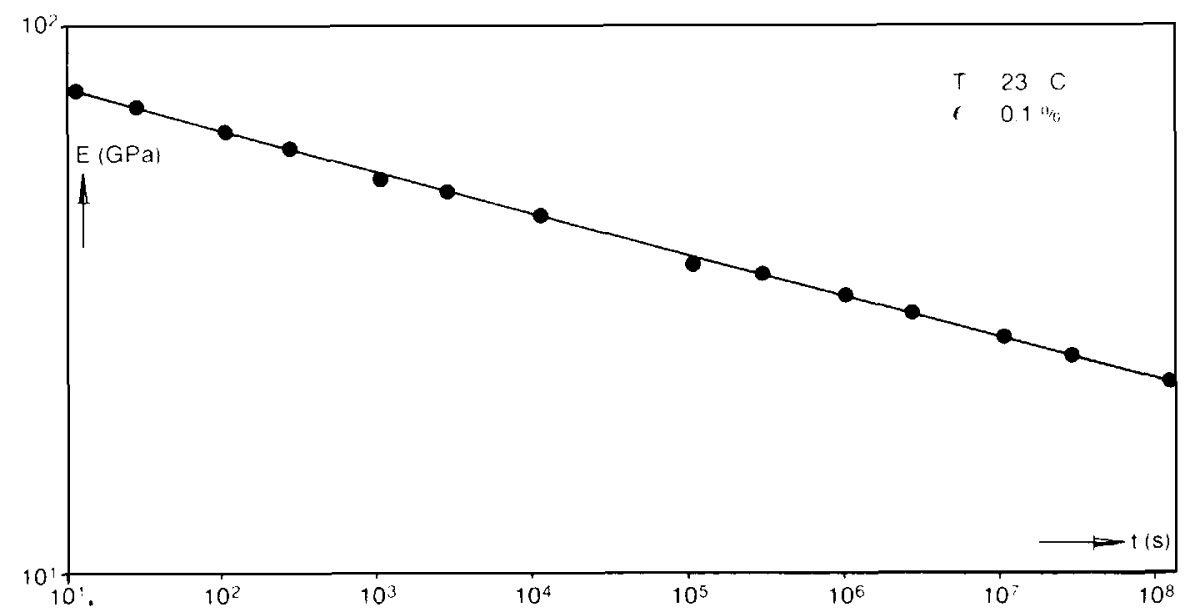

Fig. 4. Master curve of the stress relaxation modulus. Measurements at 23,50 , and $90^{\circ} \mathrm{C}$ have been combined. 


\section{DISCUSSION AND CONCLUSIONS}

As far as step strain stress relaxation, constant strain rate, and creep experiments are concerned, the viscoelastic behavior in the linear region in simple extension of the UHMW-PE fiber is in accordance with the Boltzmann superposition principle. Applying the superposition principle to the power-law modulus leads to a constitutive equation that is equivalent to that of a Maxwell model with a continuous power $n$-law spectrum. Two quantities-the prefactor $C$ and the power $n$-which can be determined easily from experimental data, allow calculation of all linear viscoelastic quantities in simple elongation. More critical tests, like dynamic measurements and recovery experiments, involving a decreasing elongational strain should be performed to further test the equation. The observation by Wilding and Ward ${ }^{5}$ that the strain is fully recoverable in the linear viscoelastic region is compatible with the equation presented here. The temperature dependence of the viscoelastic properties can be incorporated into the constitutive equation via time-temperature superposition.

Since the equation is a phenomenological one, it does not give any direct indication about the molecular processes occurring during deformation. Yet, it can also be derived from a molecular kinetic model: the temporary network model of Lodge. ${ }^{6,7}$ This model considers the material as a temporary network with junctions that are continuously destroyed and reformed while the number of junctions is constant in time. The continuous creation and destruction process is only conceivable for physical interactions like van der Waals interactions in the fiber but not for chemical interactions. Also the fact that the strain is completely recoverable indicates that the process occurring during deformation is of a physical nature.

Finally, the fact that various other materials display linear viscoelastic behavior similar to that of the UHMW-PE fiber might throw some light on the molecular processes that occur during deformation and on the kind of structures that undergo these processes.

The combination of eqs. (1) and (2) is the one-dimensional equivalent of the gel equation proposed by Winter and Chambon., ${ }^{8,9}$ They observed power law relaxation for cross-linking gels at the gel point, i.e., at the transition from the liquid to the solid state. The exponent in the power law is, however, much higher than that for the PE fiber: one-half for stoichiometrically balanced network polymers. For a deficiency of crosslinker, $n \geq 1 / 2$ is observed. No networks are known with $n<1 / 2 .^{9}$

The relaxing part of the modulus of strongly cross-linked rubbers can often be described by the power-law equation for several decades on the timescale, ${ }^{10-13}$ the power being of the order of 0.07 , but these materials also exhibit an equilibrium modulus in the time-scale of measurement.

\section{References}

1. L. Boltzmann, Ann. Phys. Chem. Ergänz., 7, 624 (1876).

2. N. J. Capiati and R. S. Porter, J. Polym. Sci. Polym. Phys. Ed, 13, 1177 (1975).

3. J. D. Ferry, Viscoelastic Properties of Polymers, John Wiley \& Sons, New York, 1980.

4. J. Bischoff, E. Catsiff, and A. V. Tobolsky, J. Am. Chem. Soc., 74, 3378 (1952).

5. M. A. Wilding and I. M. Ward, Polymer, 19, 969 (1978).

6. A. S. Lodge, Trans. Faraday Soc., 52, 120 (1956). 
7. A. S. Lodge, Rheol. Acta, 7, 379 (1968).

8. H. H. Winter and F. Chambon, J. Rheol., 30, 367 (1986).

9. F. Chambon and H. H. Winter, J. Rheol., 31, 683 (1987).

10. T. L. Smith and R. A. Dickie, J. Polym. Sci. A-2, 7, 635 (1969).

11. J. G. Curro and E. A. Salazar, J. Appl. Polym. Sci., 19, 2571 (1975).

12. S. D. Hong, R. F. Fedors, F. Schwarzl, J. Moacanin, and R. F. Landel, Polym. Eng. Sci, 21, 688 (1981).

13. G. Marrucci and B. de Cindio, Rheol. Acta, 19, 68 (1980).

Received May 17, 1988

Accepted September 7, 1988 